\section{Niveau intellectuel et capacités langagières des enfants atteints d'amyotrophie spinale de type 1}

\section{Résumé}

Le but de l'étude citée en référence [1] est d'évaluer les fonctions cognitives et langagières d'enfants atteints d'amyotrophie spinale de type 1 (SMAl). Pour cette forme, qui est la plus sévère des SMA, aucune étude n'avait pour l'instant été réalisée dans ce domaine. Dans la SMAl, outre d'importants dysfonctionnements bulbaire, respiratoire et moteur périphérique, les troubles moteurs de la sphère oro-pharyngée touchent l'élocution du langage. Celui-ci est peu fluide et peu compréhensible. La communication s'effectue essentiellement par des mouvements oculaires, des sons gutturaux. Au niveau du langage verbal, sont notés anarthrie, dysarthrie, voix nasonnée et dyslalie. II s'agit d'une étude rétrospective incluant 22 enfants atteints de SMAl (10 atteints d'un sous-type la-lb: $A B$ et 12 par lc: C) âgés de 3 à 11 ans dans un état cliniquement stable. Ils pouvaient communiquer en oui-non selon un codage. Les domaines suivants ont été évalués : niveau cognitif ( $\mathrm{Pl}$; test de Raven unidimensionnel, RCPM) ; troubles de la parole (ALS Severity Score, ALSSS) ; compréhension morphosyntaxique du langage (Brown Bellugy, TCGB) ; atteintes neuromusculaires (test infantile de troubles neuromusculaires de I'hôpital pour enfants de Philadelphie, CHOP-INTEND). Le $\mathrm{QI}$ médian est de 120 quel que soit le type de SMAl ( $A B$ ou $C$ ). La compréhension morphosyntaxique est normale. L'atteinte de la parole est à 5 (échelle de 1 [non vocal] à 10 [parole normale]), elle est corrélée au niveau d'atteintes neuromusculaires (scores CHOPINTEND) mais pas aux $\mathrm{OI}$ ni au niveau de compréhension morphosyntaxique (reconnaissance des mots, de leur terminaison, de leur agencement dans une phrase...).

Conclusion: le niveau cognitif global se situe dans la plage normale et même légèrement supérieure de la

\section{Lu pour Vous}

\section{Clinique}

Christian Réveillère ${ }^{1-3}$

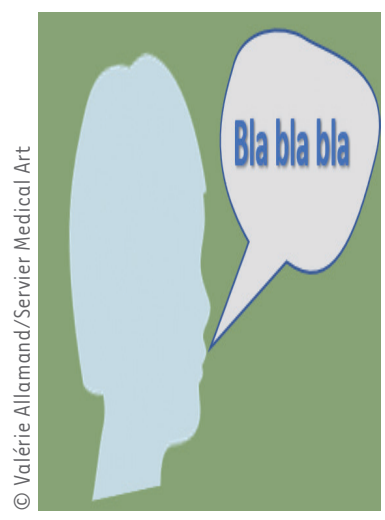

${ }^{1} A F M$-Téléthon, Évry, France. ${ }^{2}$ Centre de Référence Maladies Neuromusculaires, CHU Henri Mondor, Créteil, France.

${ }^{3}$ Université de Tours, France. creveillere@afm-telethon.fr

population générale, la compréhension du langage est préservée, seule l'atteinte motrice oro-pharyngée induit le déficit de parole et entrave le langage verbal sur un plan expressif.

\section{Commentaire}

Cet article est important [1] car il complète un autre article de revue systématique de la littérature de 2019 traitant de ce même thème «SMA et cognition » et qui mentionnait que «si la cognition semble bien préservée dans la SMA de type II et III, avec parfois des observations d'enfants hyper-performants, le doute subsiste pour la SMA de type I »[2]. Effectivement, les données étant manquantes dans la SMAl, il est très intéressant de constater qu'en dépit d'un manque d'interactions neuro-motrices avec l'environnement, le niveau intellectuel de ces enfants lourdement handicapés se développe normalement et que la compréhension du langage n'est pas non plus affectée (et ce, quel que soit le type de SMAl). Seule la parole est, de manière indépendante, touchée par le déficit neuro-musculaire. Cet article renseigne sur l'histoire naturelle de cette maladie cliniquement homogène, et fournit des repères cognitifs et langagiers pour les essais cliniques médicamenteux qui pourraient modifier la trajectoire clinique de la SMAl. La qualité de la parole dépendant de la gravité la maladie, il est en effet possible d'espérer qu'une efficacité sur la symptomatologie neuro-motrice et oro-pharyngée améliore la performance de la parole.

Concernant la vie au quotidien et la prise en charge de ces enfants avec de lourds handicaps physiques et de parole, ces premières connaissances sont utiles pour prévenir le risque de sous-estimer les capacités cognitives et de compréhension langagières. Ces dernières demandent à être stimulées et entretenues car elles sont positivement associées aux apprentissages, à la participation active à l'école, en 
situation de vie sociale, familiale..., et cela ne peut qu'être favorable à la qualité de vie des ces enfants. Du côté des parents, leurs perceptions et émotions sont forcément affectées par le caractère très visible des déficits neuro-moteurs et de parole, et leurs conséquences en termes de dépendance. Cela ne peut être que soutenant pour eux de leur communiquer les résultats des évaluations de ces domaines cognitifs qui sont préservées. II sera aussi important de leur donner des conseils de communication verbale et non verbale et dans ce dernier domaine, de penser à des modes de communication alternative. $\diamond$ Intellectual skills and speech abilities in children with SMA type 1
LIENS D'INTÉRÊT

L'auteur déclare n'avoir aucun lien d'intérêt concernant les données publiées dans cet article.

\section{RÉFÉRENCES}

1. Zappa G, Lo Mauro A, Baranello G, et al. Intellectual abilities, language comprehension, speech, and motor function in children with spinal muscular atrophy type 1.J Neurodev Disord $2021 ; 13: 9$. doi: 10.1186/s11689-02109355-4

2. Polido GJ, de Miranda MMV, Carvas N, et al. Cognitive performance of children with spinal muscular atrophy: a systematic review. Dement Neuropsychol $2019 ; 13: 436-43$

\section{wWw.myobase.org}

Catalogue en ligne disponible gratuitement sur Internet publié par l'AFM-Téléthon.

Retrouvez facilement toutes les références bibliographiques sur les maladies neuromusculaires, les situations de handicap qu'elles génèrent et leurs aspects psychologiques.

\section{Myobase donne un} accès libre à $75 \%$ du fonds documentaire collecté depuis 1990, représentant plus de 40000 références spécifiques du domaine des maladies neuromusculaires.

$>$ articles de la littérature biomédicale et psycho-sociale

\section{$>$ livres, thèses}

$>$ guides d'associations et rapports institutionnels d'agences internationales

$>$ brèves en français, synthèses des articles médico-scientifiques internationaux les plus pertinents

\section{$>$ publications}

AFM-Téléthon destinées aux professionnels de santé ou aux personnes atteintes de maladie neuromusculaire et à leur entourage

\section{UN OUTIL ERGONOMIQUE, UNE INTERFACE BILINGUE}

\section{- Laissez-vous guider par les tutoriels}

- Lancez une recherche et affinez votre sélection grâce aux filtres

\begin{tabular}{|c|c|}
\hline \multicolumn{2}{|c|}{ TOUT MYOBASE } \\
\hline \multicolumn{2}{|c|}{ Rechercher... } \\
\hline \multicolumn{2}{|c|}{ Recherche avancée } \\
\hline \multirow[t]{3}{*}{ Histol } & FILTRES \\
\hline & Type de document \\
\hline & $\begin{array}{l}\square \text { Article [3443] } \\
\square \text { Publication AFM [176] } \\
\square \text { Thèse/Mémoire [107] } \\
\square \text { Brève [102] }\end{array}$ \\
\hline
\end{tabular}

\ PUBLICATIONS AFM-Téléthon

- BRËVES

• DOCUMENTS DE SYNTHÈSE

INSTITUT DES BIOTHÉRAPIES PUBLICATIONS

- Partagez les résultats de votre recherche
UN ACCÈS facile et simple

Rechercher avec des opérateurs :

- guillemets pour une expression

"maladie de pompe"

- + pour signifier ET, et retrouver tous les documents contenant les deux mots "fauteuil +électrique"

- - pour signifier NON et enlever le mot de la recherche :

"autonomie -établissement"
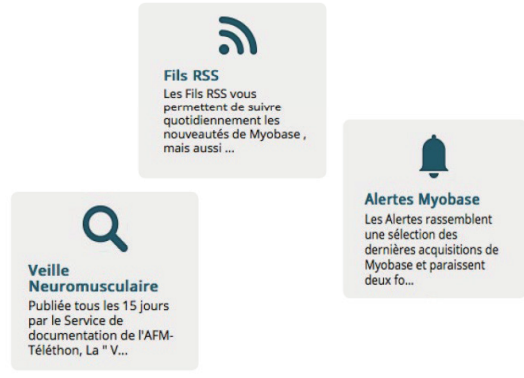

- Cliquez sur l'onglet thématique qui vous convient (haut de la page d'accueil)

- Créez vos alertes personnalisées en ouvrant un compte personnel

- Téléchargez la Veille Neuromusculaire

- Abonnez-vous aux flux RSS 